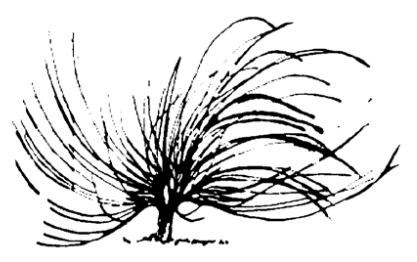

\title{
Las lagunas discursivas en las producciones de un diario de campo y de un portafolio: ¿Pérdida de sentido de lo que se enseña?
}

\author{
Ariadne Catarine dos Santos ${ }^{1}$ \\ Universidad de São Paulo \\ Brasil \\ ariadne.catarine@gmail.com
}

\begin{abstract}
Lexy Medina Mejía ${ }^{2}$
Universidad Pedagógica Nacional Francisco Morazán

Honduras

lexymedinamejia@gmail.com
\end{abstract}

\begin{abstract}
Resumen
En los últimos años, muchas investigaciones académicas en el área de estudios de la lengua (Barzotto y Eufrasio, 2009; Fairchild, 2013, 2014; Rezende, 2010) han demostrado que la práctica docente debe ser pensada como un acto pedagógico reflexivo, en el cual el estudiantado en práctica se convierta en un sujeto investigador de su propia acción, ya que ese momento permite el análisis y la evaluación de la experiencia docente a fin de validar los conocimientos, metodologías y estrategias de aprendizaje. Dentro de esta práctica, el diario de campo, como un banco de datos de la observación atenta de cada practicante, y el portafolio final, como una producción
\end{abstract}

http://dx.doi.org/10.15359/rep.esp-20-1.5

1 Profesora de lengua portuguesa y sus literaturas, revisora. Máster en Letras, en el área de los Estudios Comparativos de Literaturas en Lengua Portuguesa, por la Facultad de Filosofía, Literatura y Ciencias Humanas (FFLCH), de la Universidad de São Paulo (USP).

2 Pasante del Doctorado Latinoamericano en Políticas Públicas y Profesión Docente, Master en Currículum, Profesora de Educación Media en la Enseñanza del Español en el Grado de Licenciatura de la Universidad Pedagógica Nacional Francisco Morazán (UPNFM). 
escrita reflexiva sobre los datos observados, se convierten en los instrumentos críticos más importantes de la labor docente en el aula de clase. Las producciones escritas de estudiantes en práctica profesional de la carrera de español de una universidad pública en Centro América han demostrado que el estudiantado practicante, muchas veces, no sabe qué uso hacer de estas herramientas, por la desarticulación entre los discursos de los documentos oficiales con sus producciones discursivas, como si ocurriera una pérdida de sentido de lo que se enseña y de lo que es creado como producto final. Seleccionamos un diario de campo y un portafolio, considerado por el profesorado tutor como un excelente trabajo, para analizar cómo el estudiantado articula y analiza los datos que él mismo registró; la coherencia del discurso producido con los conocimientos específicos del área de estudios, en el caso enseñanza de español; el sentido ( o no) y la utilidad (o no) que el diario tiene para el estudiantado en práctica y para el mismo personal asesor de práctica; y finalmente, se discute el papel del profesorado tutor, en relación con el acompañamiento y supervisión de la práctica profesional.

Palabras clave: Práctica profesional; diario de campo; portafolio; lagunas discursivas; producción escrita.

\begin{abstract}
In recent years, many studies about language (BARZOTTO \& EUFRASIO (2009); FAIRCHILD (2013, 2014); REZENDE (2010)] have shown that a teaching practicum has to be thought as a reflexive pedagogical event, where students become researchers of their own teaching experience; such activity allows them the analysis and assess of their own experience and also the validation of knowledge, methodologies and teaching strategies. It is during this teaching practicum that teaching journals, as a database of the close observation of the practicum-student, should become an important reflexive instrument of classroom activity. However, the written products from Spanish teaching practicumstudents in a public university in Central America show that, on many occasions, students do not know how to
\end{abstract}


use such resource, since journals show little connection between the discourse of the official documents and the student's written work. Such problem gives the idea that the meaning of concepts taught may not be found in the final work a student submits. For the purpose of this research, we selected a journal and a portfolio from a student in a Spanish teaching program. These two sources were considered successful final productions. Moreover, they allowed the researchers to start a discussion about how this student articulated and analyzed his own data. Coherence of the produced discourse and the specific knowledge from the program - in this case Spanish teaching - the meaning ( or not) and the usefulness such productions have for the practicum-student and for the practicum-supervisor, and the role of the teacher as the one in charge of the follow-up and monitoring of the practicum are discussed.

Keywords: Teaching practices; Journal; Portfolio; Discursive gaps; Written production.

\section{CONSTRUCCIÓN DEL DIARIO DE CAMPO Y DEL POR- TAFOLIO: CAMINOS DE LA FORMACIÓN DOCENTE}

Este artículo forma parte de la investigación mayor "La escritura sobre las prácticas de enseñanza en las licenciaturas de Brasil, Costa Rica y Honduras: Registro, análisis y producción de conocimiento ${ }^{3}$ " y tiene como objetivo reflexionar sobre la importancia de la construcción de los diarios de campo y de los portafolios en las licenciaturas (carreras de formación de docentes en el nivel universitario). Para eso, esencialmente, a partir de un diario de campo y de un portafolio se discutirá cómo un profesor practicante trabaja con los datos que analiza, ya sea cómo transcribe (o no) lo que observa, reflexiona sobre su propia práctica y analiza los datos basados en los conocimientos específicos de su formación académica.

Se ha notado en los fragmentos analizados, de un diario del campo y de un portafolio de un practicante de la carrera de Letras y Lenguas

3 Llamada Universal MCTI/CNPQ No 14/2014, coordinado por el Prof. ${ }^{\circ}$. Dr. ${ }^{\circ}$ Thomas Massao Fairchild. 
- Enseñanza de Español, de una universidad pública en Centro América, que, al no saber dar un tratamiento a lo que observa, el profesor en práctica formula un discurso lacónico, lo cual no lo auxilia en la construcción de materiales didácticos significativos, aún menos refleja una formación específica en la enseñanza de una lengua, ni una visión crítica de los problemas observados en la sala de clase. Es importante resaltar que el material analizado fue considerado como un trabajo exitoso por el profesor asesor que acompañaba al estudiante en práctica.

La escritura del diario de campo y del portafolio son los instrumentos pedagógicos más importantes del profesorado durante las prácticas supervisadas. Las anotaciones del diario y los posteriores análisis de datos sirven como herramientas de reflexión para el futuro personal docente y para el resto de docentes que lo acompañan como responsables de la práctica. La escritura actúa como una versátil herramienta potencializadora (Rezende) tanto para que el alumnado pueda reflexionar sobre lo que observa en el ambiente escolar (y así hacer la articulación con el abordaje de los contenidos de su área específica) como para que el profesorado asesor pueda analizar la manera en que quien realiza la práctica docente está articulando lo que ha aprendido en la universidad con lo que ha observado en la realidad; y, de esa forma, pueda actuar de manera crítica en relación con la estructuración de la licenciatura y lo que se enseña en el curso:

Transformados respectivamente en género académico (en la licenciatura) y en género escolar (en las escuelas de educación infantil y educación básica), el relato y el proyecto pueden ser potencializados como objetos de reflexión e ir más allá de un mero ejercicio técnico, resistiendo al simplismo y a la banalización que sufren cuando se tornan actividades "obligatorias". Esos géneros, cuando son producidos en la universidad, pueden significar la posibilidad de reflexionar - ya que existe una dimensión metalingüística inherente a la escritura en los cursos de Letras sobre esas modalidades (por otra parte, hay quienes defienden que toda escritura es metalingüística, o sea, ayuda a reflexionar sobre la propia lengua, una vez que es preciso hacer elecciones en el interior de la lengua). (Rezende, 2010, pp. 47 -48) 
Se espera que el profesorado en práctica, a través de la "incorporación y la operacionalización de los conocimientos específicos ofrecidos en la formación en Letras/ Licenciatura" (Barzotto, Eufrásio, 2009 , p. 01), produzca textos que profundicen, a partir de un análisis coherente de los datos encontrados; haga reflexiones críticas en cuanto a los problemas planteados en su observación y cree posibilidades didácticas significativas. Las anotaciones del diario, en la Licenciatura en Letras, tienen como función, en esencia, servir como corpus para que el estudiantado pueda reflexionar sobre las diversas manifestaciones lingüísticas ocurridas en el aula (interacción entre estudiantes, estudiantes entre sí, con su docente y con los textos leídos), para que así el estudiantado en práctica pueda crear sus propios modos de enseñanza, a través de un trabajo autoral y por medio de la valoración de la singularidad del aprendizaje de sus estudiantes. Es importante que el estudiantado universitario asuma una postura investigativa y no critique el trabajo observado de sus pares con juicios de valor, antes que argumente de forma crítica y sepa comprender por qué algunos métodos dan más resultados que otros.

Sin embargo, lo que se observa en los fragmentos analizados es que el profesor en práctica sabe muy poco sobre cómo hacer los registros y darles tratamiento a los datos recogidos en el aula. El estudiante observa, registra los datos y expone las actividades realizadas de manera significativa, precisa y de manera coherente, pero sin profundizar para realizar su propio análisis.

Los escritos que nos sirven como corpus se componen de informaciones vagas que poco revelan sobre cómo se realizó la práctica y cuáles fueron sus resultados; de lo que se desprenden las siguientes preguntas sobre la formación en la licenciatura:

- En cuanto a la producción textual de sus estudiantes en el final de nivel pregrado:

a. ¿Cómo escriben?

b. ¿Saben producir un texto unido y coherente?

c. ¿Articulan datos y los analizan?

- En cuanto a la construcción del diario de campo y portafolio:

a. ¿Qué es lo que se les enseña sobre escribir diarios de campo y reflexionar sobre los datos?

b. ¿Cuáles son las posturas que se esperan en la práctica profesional respecto a su escritura? 


\section{FRAGMENTACIÓN DEL CAMINO}

El diario de campo y el portafolio que se analizaron fueron escritos por un estudiante del último año de la carrera del Profesorado en la Enseñanza del Español, en el grado de licenciatura, en una universidad pública de formación docente en Centro América. La carrera tiene duración de 4 años, distribuidos en 12 períodos académicos de 13 semanas cada uno. En el último período, todo el estudiantado de la licenciatura debe elegir una institución pública del nivel secundario para realizar su período de práctica profesional.

La práctica, según el Reglamento de práctica profesional ${ }^{4}$ de la universidad, se define como un acto pedagógico reflexivo, el cual está pensado y apoyado a partir de las demandas sociales de la realidad. Es un proceso transversal del currículo, caracterizado por sus espacios de docencia, investigación, extensión y reflexión sobre su quehacer profesional, a la vez, que permite la vinculación de la universidad con las comunidades del país.

En teoría, se estructura en espacios académicos procesuales y dinámicos que permiten la acción -reflexión- acción, mediante el análisis de las experiencias docentes con un alto grado de compromiso para validar los conocimientos, las metodologías y estrategias de aprendizaje por parte del estudiantado en práctica. Así, para ejecutarse como tal, requiere de la participación de distintos actores tales como el profesorado asesor (asignado por la universidad), profesorado tutor (titular de la clase) y el estudiantado en práctica.

Además, según el reglamento de la universidad, el proceso de práctica docente debe estar compuesto de algunas producciones textuales. El estudiantado debe producir documentos descritos como: Planes de clases, estrategias de aprendizaje, secuencias didácticas, estrategias de evaluación, diario de aula, portafolio docente y el informe final de práctica profesional. Los grandes ejes didácticos en el desarrollo de la práctica profesional tales como la planificación y la evaluación representan el inicio y el cierre de la acción docente, pero entre ellos existen los procesos deliberados y creativos de quien enseña, llamados estrategias de aprendizaje, los cuales se establecen en la tipología del conocimiento como actitudinales, procedimentales y declarativos; a su

4 Reglamento de Práctica Profesional, Capítulo XII, del Reglamento del Estatuto (UPNFM, 2010). 
vez, según el reglamento, se deben interpretar en las secuencias didácticas, ya que estas describen los eventos o procesos por los que se activa la enseñanza.

La elaboración de esos documentos requiere ciertas habilidades para incluir descripciones y argumentaciones de los hechos o recuentos anecdóticos ocurridos en el aula de clase y por toda la institución educativa. Al describir en el diario de clase las secuencias didácticas se puede visualizar, si se han logrado las expectativas, cómo se ha enseñado la experiencia de aprendizaje, los posibles desafíos, entre otros; dicha información objetiva permite al profesorado asesor determinar la capacidad metacognitiva y didáctica del estudiantado en práctica.

Sin embargo, a pesar de que los documentos oficiales afirmen la importancia del diario de campo como herramienta para reflexionar sobre las etapas de elaboración de la didáctica en el aula y como documento base de la producción del portafolio, se observa que los documentos exigidos se trabajaron en la siguiente secuencia:

Figura 1: Descripción de la secuencia de documentos trabajados con estudiantes en la práctica profesional

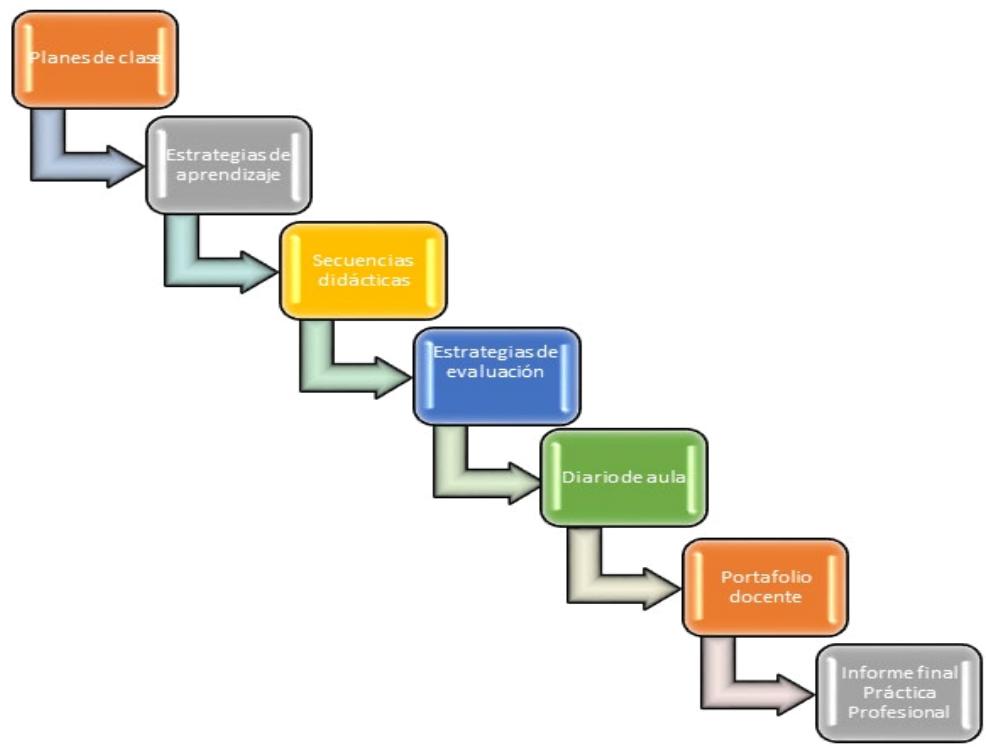

En la Figura 1, se visualiza que el diario de campo no es la primera producción hecha por el estudiantado, por lo que su función 
resulta vacía de sentido, debido a que los datos no son recogidos a lo largo de la práctica docente. Se percibe que la ausencia de información sobre lo que se ha observado en el aula dificulta la producción posterior de un portafolio reflexivo sobre la práctica docente del profesorado practicante, una vez que faltan datos por analizar.

Se puede percibir en este análisis que las anotaciones realizadas durante la práctica quedan fragmentadas en diversos documentos, los cuales muchas veces no se articulan entre sí. Además, el profesorado practicante parece no comprender la importancia de cada texto, su finalidad y la articulación entre ellos; de esa manera el estudiantado no sabe distribuir las informaciones conforme las especificidades de cada género textual o en qué momento debe recoger las informaciones de la persona que observa o en qué espacio debe analizar o cómo analizar los datos; por eso, muchas veces, las producciones textuales se tornan apenas papeles para llenar, una vez que el estudiantado quiere cumplir la recta final para graduarse.

\section{EL REVOLTIJO DE PAPELES: LAS LAGUNAS DISCUR- SIVAS EN EL DIARIO DE CAMPO Y EL PORTAFOLIO}

Entre los documentos solicitados al sujeto practicante por el reglamento de la universidad, como se visualizó antes, dos de ellos deberían tener fundamental importancia: el primero es el diario de campo, donde debe recolectar datos de su período de observación y de práctica; y el segundo es el portafolio, donde debe analizar los datos que recoge a partir de los conocimientos específicos de su área.

Cada uno de ellos forma parte de un género textual: El diario de campo está más ligado al relato instantáneo y a las características de la oralidad a través de las minuciosas percepciones de los hechos ocurridos y de las transcripciones de las conversaciones de los agentes discursivos. Como afirma Rezende (2010, pp. 47-48), el diario no aspira una unidad en cuanto a variedad de lo que es narrado, una vez que abarca: "ideas, parpadeos y percepciones registradas junto con las descripciones de las escenas escolares cotidianas y de las anotaciones objetivas y de los datos". Si el diario expresa una variedad de asociaciones, que permiten una discontinuidad entre los diversos hechos narrados, los mismos deben estar construidos con informaciones unidas y coherentes sobre lo ocurrido en el aula; esas informaciones entrelazadas deben formar un todo lógico y continuo, o sea, al correr las páginas 
se deben comprender los datos relatados para que, a partir de ellos, se pueda reflexionar y crear una práctica docente significativa.

El término portafolio presenta sus diferenciaciones, pues el portafolio debe garantizar la unidad de las ideas, proponer análisis para los datos traídos por el diario de campo y hacer articulaciones con las teorías aprendidas durante los estudios ${ }^{5}$. Este es el momento en que el profesorado practicante puede vincular la parte teórica con la metodología y la práctica. Todavía, la idea teórica de portafolio está muy distante de la presentación práctica, los portafolios encontrados sugieren la idea de una cartera para llevar libros y papeles. Alude la idea de documentos de carácter más testimonial, pero menos científico. Por eso, se utilizan los términos "informe" o "relatorio", los cuales están más vinculados a la idea de ciencia de un hacer sistemático y racionalizado.

En este artículo se analizarán algunos extractos ${ }^{6}$ del diario de campo y del portafolio de un estudiante que realizó la práctica en el segundo periodo (mayo a agosto) del año 2015. Las anotaciones del diario de campo fueron entregadas en un cuaderno pequeño, todos los apuntes fueron escritos a mano y a veces hemos tenido dificultades para entender la escritura del estudiante. El diario contiene un total de 56 páginas, con las descripciones de los 56 días de práctica profesional. El portafolio está compuesto de 459 páginas, todas digitalizadas y decorado con dibujos, las hojas se recogen en una carpeta grande, donde el estudiante hizo un ayuntamiento de los documentos exigidos en la práctica profesional.

Los relatos del diario son cortos y tienen como máximo 15 líneas; por lo que, debido a las pequeñas descripciones, se vuelve difícil situar en el discurso escrito por el profesor practicante datos básicos como: lo que sucedió en cada sección, quiénes fueron los sujetos involucrados, en cuáles grados ocurrió la práctica, cómo reaccionó el alumnado ante su presencia como profesor practicante, etc. ${ }^{7}$. Por ejemplo, en la primera página del relato del estudiante hay una descripción general sobre las clases que le fueron asignadas: Octavo grado sección 5 y 6 , noveno grado sección 2, Bachillerato Técnico Profesional en Administración de

5 Reglamento de Práctica Profesional, Capítulo XII, del Reglamento del Estatuto (UPNFM, 2010).

6 Las marcas en negrita indican algunas frases en las que nos detenemos.

7 Las transcripciones reproducen correctamente las notas, se optó por mantener a los errores de ortografía, uso de mayúsculas y minúsculas como lo estaban en el cuaderno en cuestión. 
Empresas sección 5. Sin embargo, en los demás días, no se sabe en cuál clase el estudiante está actuando.

En la descripción del primer día no hay datos suficientes para saber cuál es la sección que el alumno está observando entre las que le fueron asignadas.

\section{Fragmento 1:}

Martes, 19 de mayo de 2015

"Al arribar al colegio configuramos los horarios con los compañeros de manera que no hubiese choque en ninguna clase, posteriormente nos desplazamos a las aulas de clase para la respectiva observación, y pude percibir, el trato entre docente y alumno, el carácter que debe tener el docente para que el alumno pueda estar atento en la clase".

Además, no se tiene información sobre la cantidad de estudiantes que había ese día, ni descripción de cómo se pasó al aula ni cuáles fueron los contenidos abordados ni las reacciones de estudiantes y docentes. No hay comentarios del profesor asesor ni en cuanto a la descripción de lo observado ni en cuanto a los errores ortográficos cometidos, como en la palabra "compañeros" (escrita sin la eñe). El comentario más importante en este pasaje es "Carácter que debe tener el docente", porque es el único que tiene que ver con lo pedagógico y no con cuestiones logísticas, pero dicha expresión no informa a quien leer sobre cuál es el carácter que según el estudiante debe tener el personal docente.

Se notó también que el estudiante tuvo dificultades para organizar las descripciones por fechas, y en algunos momentos no se tiene certeza de cuántos días están siendo descritos o que sucedió cada día, por ejemplo.

\section{Fragmento 2:}

\section{"20-22 de mayo 2015}

Durante estos días adquirí mis temas para la elaboración de planes de Clase y material didáctico, colaboré con la maestra en la revisión de trabajos, así como también en el desarrollo de actividades en las que las jóvenes necesitaban ayuda."

El estudiante colocó la siguiente fecha: 20-22 de mayo de 2015, por lo que se infiere que el relato de 15 líneas comprende 2 o 3 días de observación. En las primeras líneas, el estudiante afirma que observó a 
la profesora titular para tener una idea del trato entre la docente y sus estudiantes; pero no dice lo que observó. No hay transcripciones de las conversaciones entre profesora y estudiantes. El practicante también menciona el material didáctico, planes de clase, pero no hace ningún análisis. Después afirma haber ayudado a la profesora titular con la revisión de trabajos, pero no dice qué trabajos son esos. Informa sobre la ayuda que prestó en el desarrollo de algunas actividades, pero no se sabe cuáles fueron esas actividades, mucho menos cuáles fueron las mayores dificultades del grupo de jóvenes, que él como practicante dijo haber observado.

Las presentaciones de los contenidos trabajados también se hicieron de manera lacónica como se observa en el siguiente relato.

\section{Fragmento 3:}

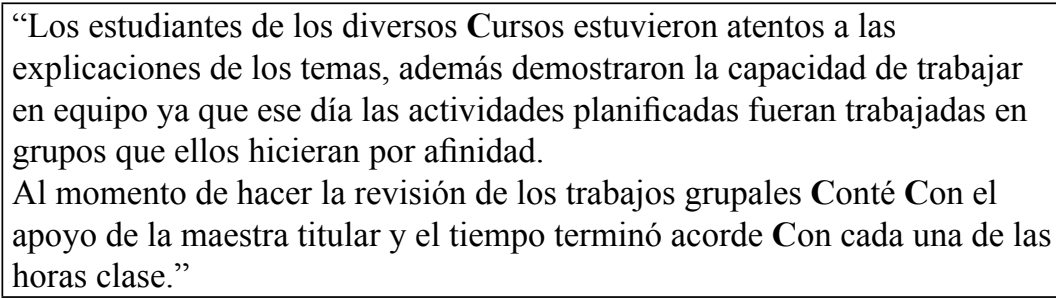

El alumno continúa haciendo referencia a algunos temas que están siendo trabajados, pero no se sabe cuáles fueron. El profesor practicante dice que el trabajo entre estudiantes aconteció en grupo; todavía no se comprende cómo ocurrió esa articulación. Se sabe que el estudiante revisó las actividades con la ayuda de la profesora titular, pero no se conoce cuáles fueron las actividades ni qué discutieron. Nuevamente, los apuntes de la profesora asesora quizás se dan en el nivel de la corrección de la caligrafía, las letras mayúsculas y minúsculas y como en las indicaciones anteriores son siempre sobre la letra "C".

Pasado un mes de su periodo de observación, el estudiante realiza una primera descripción de un contenido abordado. 


\section{Fragmento 4:}

"Jueves 4 de junio de 2015
Los estudiantes estuvieran atentos a la clase y respondieran a cada
interrogante, de igual forma hicieran preguntas para poder comprender
el tema que les estaba explicando hasta quedar claros en cuanto a los
temas, justamente tratamos la acentuación de las palabras por su sílaba
tónica, aunque al inicio estaban confundidos, al hacer ejemplos varios y
contextualizadas lograron comprender la mayoría, pero para afianzar se les
asignó bastantes ejemplos para que pudiesen practicar."

Si nota que todos los términos en negrita recubren alguna información importante que no ha sido anotada. El profesor practicante dice que junto con los temas (¿cuáles?) él tuvo que abordar la cuestión de la acentuación de las palabras y sus sílabas tónicas, pero no dice cuál fue el abordaje utilizado, el estudiante solo afirma haber utilizado ejemplos contextualizados (¿cuáles?). En la descripción siguiente hay más bien una pequeña descripción de los contenidos abordados.

\section{Fragmento 5:}

\begin{tabular}{|l|}
\hline "Lunes 8 de junio de 2015 \\
Se tuvieron que Correr algunos planes en vista que el día Viernes no hubo \\
clases, pero igual Se dio el tema de manera satisfactoria a todos los cursos. \\
El curso que me llamó la atención fue el de primero de bachillerato quienes \\
expusieron sus preguntas sobre como poder diferenciar la diferencia entre \\
Homonimia y Polisemia y tuve que abordar el aspecto de la etimología \\
de las palabras para determinar la línea tenue entre estos dos fenómenos \\
lingüísticos, desde luego con ejemplos contextualizados".
\end{tabular}

El alumno afirma que pudo seguir con los temas satisfactoriamente, pero no se sabe cuáles fueron los temas. En este momento, el estudiante expone un punto más específico de su práctica, o sea, los cuestionamientos (¿cuáles?) que ocurrieron sobre homonimia y polisemia. El estudiante dice que tuvo que buscar la etimología de las palabras para explicar la "línea tenue" entre esos fenómenos lingüísticos. Nuevamente, dice que usó ejemplos contextualizados, pero no muestra cuáles fueron los ejemplos o cuáles fueron las palabras que les causaron duda al grupo de estudiantes. 


\section{Fragmento 6:}

"Martes 9 de junio de 2015

Durante este día no se impartió clases porque estaba programada la aplicación de Central de lectura y en uno de los cursos, el desarrollo de una plenaria para la aplicación de la evaluación del libro.

Plenaria donde todos demostraron un gran interés sobre el libro leído "La Dama de las Camelias". Cada una expuso su perspectiva sobre el libro destacando la temática principal así como la caracterización de los personajes".

En esa parte del relato se torna interesante pensar cuál es la noción que el practicante tiene de "clase"; ya que, a pesar de haberse realizado una supuesta actividad, el estudiante escribió que no hubo clases. Él expresa que la discusión fue muy buena y que discutieron los aspectos de los personajes: ¿Cuáles aspectos?, ¿Cómo esa actividad fue articulada con lo que había sido enseñado anteriormente? Se vuelve a percibir la discrepancia en la recogida de datos para la escritura del relato: ¿Cómo ocurrió esa discusión?, ¿Quién participó en la misma? ¿Por qué eso no fue considerado como una clase?, ¿De qué manera fue abordado el libro?, ¿El libro fue contextualizado?

\section{Fragmento 7:}

"Miércoles 10 de junio de 2015

Hubo suspensión de clases con la mayoría de los Cursos debido a que ese día se programó una película para todos los estudiantes".

En un relato corto, el profesor practicante nuevamente dice que no hubo clase, porque se programó una película. Eso es porque él considera clase como siendo "exposición de contenido + ejercicios". Quizás él niega que el debate sobre el libro o la película sean "clases", porque no quedó claro cómo el contenido de esas actividades tiene que ver con la lengua española. De haber hecho mejores apuntes sobre qué sucedió en esas ocasiones, pudiese haberlo tomado como tema de análisis.

Lo restante del relato continúa de la misma forma, son descripciones cortas y con pocas informaciones sobre lo que fue observado en clase y cuáles estrategias y contenidos fueron adoptados. No solamente las observaciones del estudiante no son puntuales, sino también los apuntes / los comentarios del profesor supervisor; ya que aparecen en 
pocos momentos y no interrogan las lagunas del discurso del estudiante, pues en esas anotaciones solamente se hacen observaciones sobre uso de minúsculas y mayúsculas. Muchas veces, no hay correcciones por parte del profesor asesor ni en cuanto al contenido ni correcciones gramaticales, a pesar del error ortográfico en las palabras, como muestra la imagen siguiente, Figura 2.

Figura 2: Imagen del diario de campo con los apuntes del profesor tutor

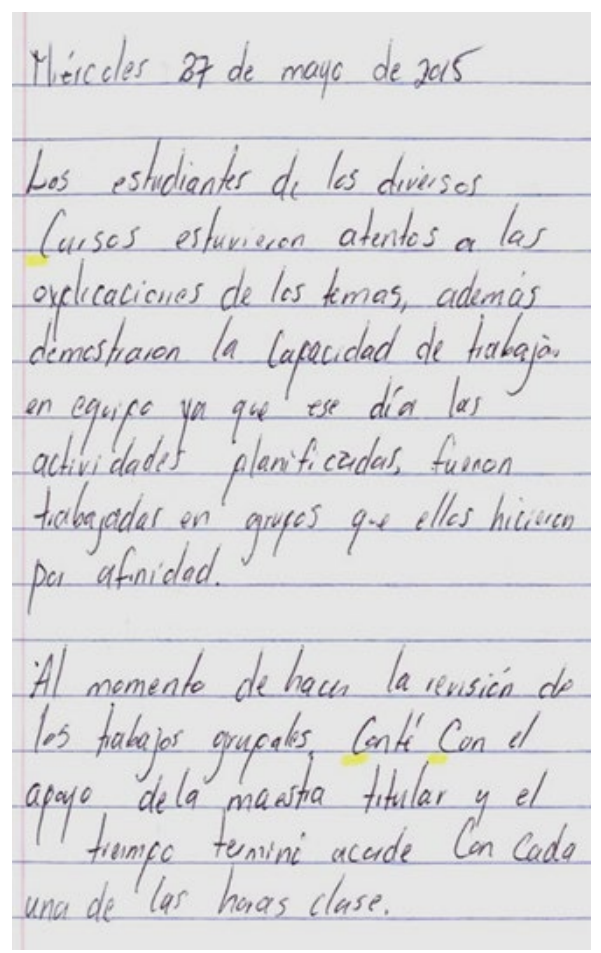

Teniendo como base que en el diario de campo se deben destacar las evidencias del desempeño del estudiantado en práctica, pues hace prevalecer en su tipología la capacidad de análisis, de reflexión y el compromiso con su propio aprendizaje, nos preguntamos cómo el profesor practicante en cuestión desaprovechó de su momento en la escuela para recolectar datos significativos que no solamente relaten aspectos de la escuela, sino que estén relacionados con su formación 
específica en la enseñanza de lengua española. Según Bolívar (2010), los diarios de campo deben incluir: a) contexto y ambiente de la clase (dinámica, relaciones sociales, participación, etc.) b) actuación del profesor o profesora (estrategias metodológicas, formas de interacción, propósitos, etc.), y c) comportamientos del alumnado (implicación en las actividades, estrategias, incidentes, etc.).

Sin embargo, se percibe que ese diario de campo, a pesar de ser considerado como un trabajo exitoso está distante de lo que la teoría y el reglamento de la universidad proponen. Desplazado de su lugar inicial y no vinculado a los demás documentos exigidos para la práctica profesional, el diario está compuesto por informaciones vagas, que solo parece un montón de papeles. Por medio de esas descripciones no se sabe cómo los asuntos fueron articulados, ni mucho menos cuáles fueron los ejemplos utilizados para explicar la acentuación y las diferencias entre homonimia y polisemia. De la misma forma, el uso del libro La dama de las camelias aparece descontextualizado, no se sabe en qué momento el estudiante consideró importante usar el romance, ni cómo se dio la selección y tampoco cómo la lectura del libro fue articulada con los otros contenidos previos. Partiendo de una visión tradicional de aula, el profesor practicante también afirma que en un día "no hubo clase" y que los alumnos asistieron a ver una película, o sea, para él, la producción cinematográfica no fue utilizada como un recurso didáctico, ni mucho menos fue puesta dentro de una secuencia.

Las anotaciones del profesor asesor también no abren posibilidades de caminos a seguir; ya que el asesor no cuestiona el texto del alumno, no hace crítica y no corrige la ausencia de información, por lo que sus anotaciones se restringen al uso de las letras mayúsculas y minúsculas, deja pasar hasta errores de acentuación y de ortografía. Con esas lagunas, el diario de campo parece apenas un papel en el que el profesor practicante no sabe qué llenar, cuáles datos debe colocar, de la misma forma que para el profesor asesor parece apenas un papel más para "dar un visto bueno".

El portafolio fue el último documento entregado por ese alumno, en una carpeta están 459 páginas separadas en ocho segmentos:

1. Reseña histórica de la escuela dónde fue realizada la práctica: No sabemos si esa reseña, con los datos de la escuela, fue escrita por 
el estudiante o si él copió el texto de algún lugar, una vez que la apariencia del texto parece una copia de un libro.

2. Hoja de vida del estudiante: Contiene los datos personales y profesionales del estudiante. Además, el estudiante contesta 15 preguntas, no sabemos si las mismas fueron hechas por el propio estudiante o por el profesor asesor.

a. ¿Qué es para mí enseñar?

b. ¿Para qué enseño?

c. ¿Cómo enseño?

d. ¿Qué es para mí aprender?

e. ¿Para qué aprendo?

f. ¿Para qué aprenden mis alumnos?

g. ¿Cuáles son las asignaturas que más me gusta enseñar? ¿Por qué?

h. ¿Qué es para mí evaluar?

i. ¿Para qué evalúo a mis alumnos? ¿Cómo evalúo?

j. ¿Cuál es el rendimiento académico de mis alumnos?

k. ¿Cuáles son mis cualidades y fortalezas como docente?

1. Matriz: ¿Cómo me enseñaron? ¿Cómo he enseñado?

m. ¿Qué me falta por cambiar e innovar?

n. ¿Cómo lo voy a lograr?

o. Soy un docente innovador porque: ¿Cuál enfoque utilizo? ¿Cómo gestiono el tiempo en el aula de clase? ¿Cómo organizo el salón de clase? ¿De qué manera controlo el grupo de alumnos?

3. Los horarios de clase que el estudiante acompañó: compuesto por dos tablas con los horarios de las clases.

4. Planes de clase: Separados en tablas están los planes de clases, las tablas son divididas en estrategias de enseñanza, estrategias de aprendizaje, recursos, estrategias de evaluación, duración, bibliografía básica e Imprevistos.

5. Evaluaciones del profesor tutor y asesor: Compuesto por una serie de tablas donde se debe analizar la actitud profesional, relación maestro-alumno, aspecto científico, aspecto técnico- metodológico. El estudiante es evaluado con una nota de 0 a 5 o indicadores como excelente, muy bueno, bueno y necesita mejorar.

6. Hojas de trabajo: Reúne todos los materiales usados por el estudiante, ejercicios, textos seleccionados, etc. 
7. Exámenes: Reúne todos los exámenes aplicados por el estudiante en el aula de clase.

8. Actividades hechas por los alumnos en clase: Reúne copias de las actividades hechas por los estudiantes en clase, resultado del trabajo, etc.

Como se ha expresado anteriormente, el portafolio elaborado por el estudiante es una carpeta para separar los papeles que evidencian su experiencia, pero no hacen una reflexión crítica sobre la práctica docente. Muchas de las informaciones que no encontramos en el diario del campo las localizamos en el portafolio, aunque todavía son informaciones breves que parecen no indicar que debemos procurar las respuestas en más lugares, o sea, son tantos documentos que cada dato está en espacios diferentes que no se comunican entre sí.

En la sección 2 del portafolio donde el estudiante contesta una serie de preguntas, las cuales no sabemos si fueron elaboradas por el estudiante o por el asesor, encontramos respuestas muy vagas, por ejemplo, cuando cuestiona sobre: “¿Para qué evalúo a mis alumnos?, ¿Cómo evaluó?", tenemos la siguiente respuesta:

\section{Fragmento 1:}

¿Para qué evalúo a mis alumnos? ¿Cómo evaluó?

Evalúo para saber el nivel de aprendizaje que han adquirido mis estudiantes y este proceso lo hago mediante variedad de metodologías y téenicas que activen la espontaneidad del educando.

De forma genérica el estudiante contesta la pregunta a través de una respuesta bien formulada que, a pesar de lo escrito, dice poco. No sabemos, por ejemplo, qué tipo de evaluaciones son elegidas, cuáles son las variedades de metodologías y técnicas utilizadas y no podemos comprender qué significa activar la espontaneidad del estudiantado por medio de la evaluación. El estudiante no se basa en su experiencia práctica para contestar la pregunta.

En otra cuestión del mismo formulario percibimos que, así como miramos en el diario de campo que el estudiante tenía una visión tradicional de clase, como lo es la "exposición de contenido + ejercicios", y por eso no consideraba la exposición de una película como una clase, 
también miramos que la relación docente-estudiante está basada en conceptos de la pedagogía tradicional en la que el personal docente es el proveedor del conocimiento y lo transmite a sus estudiantes. Para el estudiante en práctica, el profesorado debe tener una vocación para enseñar, además la escuela debe tener una formación moral que moldee el comportamiento del alumnado ante la sociedad.

\section{Fragmento 2:}

a) ¿Qué es para mí enseñar?

Considero que la enseñanza es el acto de transmitir conocimientos nuevos a otras personas $(\ldots)$

b) ¿Cuáles son mis cualidades y fortalezas como docente?

En cuanto a mis cualidades creo que la más importante es la vocación por querer transmitir el aprendizaje (...)

c) ¿Para qué aprenden mis alumnos?

Mis alumnos aprenden para ser personas de bien con el afán de encontrarse con un futuro amplio y lleno de oportunidades para su desenvolvimiento y aporte a la humanidad (...)

Si enfatiza que las respuestas dadas a las preguntas del portafolio de la práctica profesional no hacen referencia a la propia experiencia de la práctica relatada, las preguntas son contestadas de forma genérica y traen bastante de la visión del estudiante sobre qué es ser docente, la relación profesorado-alumnado y la función de la escuela. Miramos que el profesor en práctica dice que la enseñanza es una vocación, refiriéndose a la visión tradicional de la pedagogía, pero ese es un mito muy combatido, ya que la docencia no es un don, o una capacidad innata, es una carrera que debe tener una formación sólida y sus derechos garantizados (condiciones dignas de trabajos, salarios, etc.).

La visión pedagógica tradicional de que el profesorado debe transmitir conocimientos también aparece en la respuesta: "la enseñanza es el acto de transmitir conocimientos nuevos a otras personas", esa idea muchas veces puede desconsiderar los conocimientos previos de estudiantes. Paulo Freire, en Pedagogía del oprimido (1994), expresa 
que la transmisión del conocimiento no debe ser una práctica estática y vertical en que el profesorado es la figura detentora del saber; antes el aprendizaje debe ser un proceso dinámico y horizontal entre profesorado y alumnado en torno de la producción de conocimientos. De esa forma, el saber no es un producto acabado y formal, la escuela debe ser un lugar de la diversidad de saberes y respeto al prójimo, luego también es necesario reflexionar sobre el ambiente escolar como formador de moralidad, una vez que es importante respetar las diferencias e incentivar al estudiantado a pensar, no restringir su modo de ser o juzgarlo, por ejemplo, no sabemos qué quiere decir el estudiante en práctica con el enunciado "personas de bien".

Otras lagunas discursivas igualmente son encontradas a lo largo del portafolio, encontramos el plan de clase bien genérico que no expresa el tema tratado, las preguntas hechas por el docente, el mapa conceptual usado, etc., tenemos las informaciones más precisas sobre el tiempo invertido en cada etapa de clase y la bibliografía básica. Sigue la transcripción de una parte de la tabla de plan de clase. 


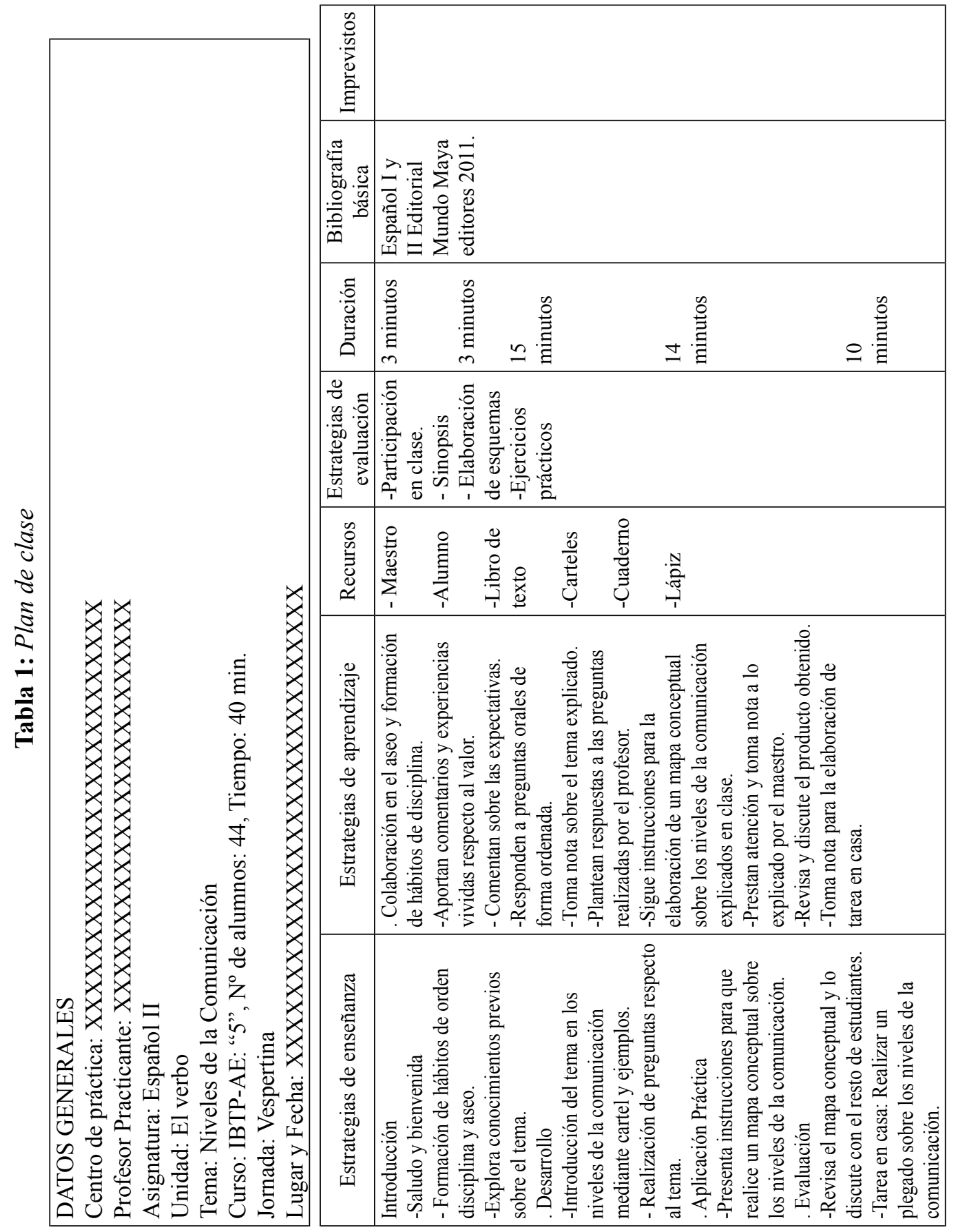


La evaluación del profesorado tutor también es en una tabla que analiza los siguientes aspectos: presentación, puntualidad, respeto a los demás y la jerarquía, responsabilidades, entusiasmo frente a estudiantes, actitudes, creatividad e iniciativas, con conceptos como "excelente, muy bueno, bueno, necesita mejorar". En el espacio "otras observaciones" el profesorado tutor a veces hace algún comentario corto sobre la actuación de cada estudiante en práctica y le felicita. Notamos que hay comentarios más específicos sobre la práctica en sí, normalmente son comentarios deseando éxitos a la carrera docente del futuro profesor.

Tabla 2: Tabla de evaluación de los profesores tutores: Rendimiento del profesor practicante

ACTITUD PROFESIONAL

\begin{tabular}{|c|c|c|c|c|c|}
\hline $\mathbf{N}$ & ASPECTOS A EVALUAR & $\mathbf{E}$ & MB & B & NM \\
\hline 1. & Presentación personal apropiada. & $\checkmark$ & & & \\
\hline 2. & Trata respetuosamente a los demás. & $\checkmark$ & & & \\
\hline 3. & Asistencia puntual a su jornada de práctica. & $\checkmark$ & & & \\
\hline 4. & Entusiasmo, participación y dedicación. & $\checkmark$ & & & \\
\hline 5. & Responsabilidad en el cumplimiento de funciones y tareas. & 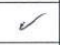 & & & \\
\hline 6. & Entrega puntual de las tareas asignadas en cada etapa. & $\nu$ & & & \\
\hline 7. & Realiza las tareas solicitadas y las actividades planificadas. & $\gamma$ & & & \\
\hline 8. & $\begin{array}{l}\text { Relaciones personales y formas de comunicación } \\
\text { adecuadas. }\end{array}$ & $\checkmark$ & & & \\
\hline 9. & Respeta la opinión de los miembros del equipo de trabajo. & $\gamma$ & & & \\
\hline 10. & Respeta la opinión de los profesores tutores. & $\nu$ & & & \\
\hline 11. & Mantiene un interés por el diálogo. & $\zeta$ & & & \\
\hline 12. & Respeto al profesor tutor. & $\nu$ & & & \\
\hline 13. & Respeto a sus compañeros de práctica. & $\zeta$ & & & \\
\hline 14. & Participación en actividades de grupo. & $\zeta$ & & & \\
\hline 15. & Interés por estimular a los alumnos. & $\checkmark$ & & & \\
\hline 16. & Actitud para trabajar en equipo. & $\gamma$ & & & \\
\hline 17. & Muestra autocontrol. & $\zeta$ & & & \\
\hline 18. & Iniciativa y creatividad. & 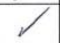 & & & \\
\hline
\end{tabular}


Figura 4: Comentario realizado por el profesor tutor sobre el desempeño del profesor practicante

\section{Otras observaciones}

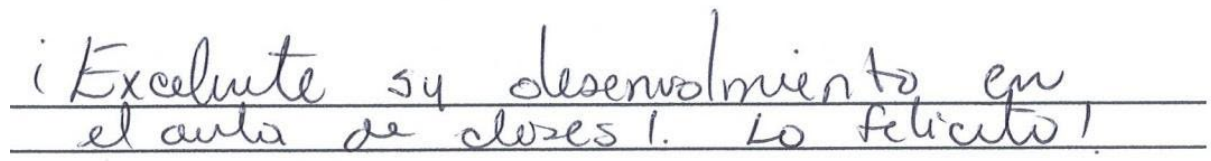

La evaluación del profesor asesor también es hecha por medio de una tabla, en la cual se analizan cuatro aspectos: actitud profesional, relación docente-estudiante, aspecto científico y aspecto técnico- metodológico. Esos aspectos están divididos en 29 cuestiones que son evaluadas de 0 a 5 puntos.

La última parte del portafolio está compuesta por los materiales utilizados en clase, algunas listas de ejercicios, algunos textos teóricos y ciertos ejemplos de trabajos hechos por los grupos adolescentes en clase.

En este artículo, en el que se analiza solo un diario de campo y un portafolio, percibimos que esto representa la media de otros diarios y portafolios finales que también contienen lagunas discursivas sobre lo que fue observado en la clase y lo que fue planificado como actividades de práctica docente y la reflexión sobre la práctica. Es necesario pensar en las varias dimensiones del problema. En una primera dimensión se mira la desorientación del estudiante particular que parece no saber o qué hacer con los datos que observa, pero la problemática no ocurre solamente en el nivel individual, por parte del estudiantado, por la lectura de otros diarios de campo percibimos que hay una mayoría que también sigue un modelo y está reproduciendo algunas otras prácticas que mira.

En otra dimensión se observa las ausencias de anotaciones del profesor asesor de este caso, que no debate ni acompaña al estudiante, ni ayuda lo ayuda a construir un camino de reflexión. Las lagunas discursivas en estos dos discursos muestran problemas en una tercera dimensión, en la cultura escolar, incluyendo la cultura universitaria, que no estimula las producciones escritas en el nivel científico, con análisis de datos y cuestionamientos, ni la propia escuela es transformada en un espacio de producción de conocimientos científicos sobre las propias prácticas docentes. En esa tercera dimensión se observa que también hay una imagen de profesorado propagada, principalmente, por la 
universidad, la cual ejecuta con más acciones impuestas lo que decide sobre la propia práctica docente.

\section{LAS LAGUNAS DISCURSIVAS Y LA PÉRDIDA DEL SEN- TIDO DE LO QUE SE ENSEÑA}

Barzotto y Eufrasio (2009), en el artículo "El informe de práctica docente como manifestación del perfil profesional en letras", en el cual también analizan la construcción de los diarios de campos en el nivel universitario, afirman que no son presupuestos que la práctica docente y la escritura del diario van a tornarlas como actividades esencialmente formadoras, principalmente si ellas no fueron pensadas como tales. La ida a las escuelas, el acompañamiento de las clases, el período de práctica y las anotaciones de los datos se tornan irrelevantes cuando pierden su sentido y cambian a metas burocráticas académicas.

La experiencia muestra que el contacto cotidiano en la escuela y sus consecuentes descripciones no hacen de la práctica una actividad esencialmente formadora, una vez que no incentivan y reafirman el perfil profesional esperado durante la formación en la licenciatura. La aceptación de una descripción general de los hechos presenciados como trabajo final de la práctica docente no exige del estudiante una postura más activa en cuanto observador y profesional capaz de intervenir futuramente en las situaciones escolares para hacer valer los conocimientos que le fueron presentados durante su formación. Eso se agrava cuando el relato se aproxima más a un llenado de fichas o una búsqueda de respuestas a cuestiones formuladas previamente, que acaban por condicionar la visión del profesor practicante para puntos específicos durante la observación, dejando escapar otros que se podrían mostrar más importantes en la situación concreta de clase. (Barzotto y Eufrásio, 2009, p. 03)

Además del llenado de metas y papeles, el estudiantado debe ser incentivado a ir a las escuelas con una visión abierta para lo que va a observar, o sea, sin juzgamientos previos, pero también debe tener una visión determinada que contemple, principalmente, su área específica de estudio. Tratándose de un estudiante de letras se espera que él observe los fenómenos lingüísticos, las producciones orales y escritas 
que se realizan constantemente en el ambiente escolar, las didácticas utilizadas, etc., y sepa articularlo con los contenidos que aprendió en las asignaturas de fonética y fonología, lingüística textual, crítica literaria, entre otras, y, de esa forma, reflexionar sobre su práctica docente. Caso contrario, la crítica que se hace sobre el distanciamiento de la universidad con la realidad de las escuelas continuará siendo la misma.

Cuando analizamos un diario de campo y un portafolio fragmentado, nos podemos cuestionar en cuanto a la producción textual del alumnado al final de su carrera. Los extractos que analizamos, por ejemplo, carecen de atención del sujeto interlocutor discursivo, la producción textual está cerrada en sí misma, o sea, el profesor practicante no alcanza a pasar a la otra persona lo que observó, no torna el conocimiento compartido.

Con eso estoy afirmando que el profesor es responsable por producir, no solamente su propia aula, sino también un conocimiento sobre el aula que no puede tener validez únicamente personal, para su propio crecimiento en la profesión, sino que es necesario adquirir valor de cambio, de forma que pueda tornarse importante también para otros. (Fairchild, 2014, pp. 01-02)

Parece que hay una pérdida de sentido de lo que se enseña, donde el discurso de los documentos oficiales de la universidad no hace más lazo con el discurso del profesorado ni estudiantado. O sea, no hay una comunicación discursiva entre lo que se espera y lo que se produce en la práctica. Las lagunas discursivas demuestran un ruido en la comunicación, en donde nadie se escucha y por eso todo parece muy fragmentado y desordenado, a pesar del supuesto orden:

Si esto de facto ocurre, tratase de un problema muy grave, pues una ruptura en la cadena de los significantes resulta en la pérdida del lazo discursivo que la escuela mantiene con aquellos la quién atiende ... Si esa cadena se rompe, la consecuencia natural es que el qué se presenta como un saber parezca sin sentido- tanto para quién escucha cuanto para quién dice. (Fairchild, 2013, p. 74)

Podemos proponer algunas hipótesis para esa situación: 
a. El discurso producido por los documentos oficiales con su exigencia sobre la producción de diferentes materiales (diario de campo, portafolio, plan de clase, etc.) está demasiado fragmentado, tanto que ni el profesorado asesor ni el estudiantado consiguen juntar las piezas del rompecabezas.

b. Frente a todos los problemas enfrentados en la realidad de la escuela pública, el profesorado asesor no mira el sentido en la producción de los documentos exigidos por las instituciones y no consiguen estimular a sus estudiantes en el proceso de escritura de cada parte del trabajo.

c. El estudiantado de letras tiene una formación inicial débil y no consigue diferenciar, en el momento final de su carrera, las características de cada género textual exigido, mucho menos consigue interpretar los requisitos y dar un significado para su producción.

d. ¿O será que el discurso lagunar resulta de una mezcla de todas las alternativas anteriores?

Por ende, si la universidad es el lugar de la producción de conocimiento, nos debemos preguntar cuál conocimiento estamos enseñando para que nuestro alumnado produzca, como está produciendo, a partir de cuáles materiales y con quién están compartiendo. La formación docente exige que el profesorado reflexione sobre la propia práctica constantemente y haga de ella objeto de estudio y discusión. De la misma forma que se aprenden diversas teorías, se aprende a ver, analizar y escribir; enseñar esos pasos se torna función principal del profesorado asesor.

Siendo así, se nota que hay una serie de factores que necesitan ser repensados cuando se habla de práctica profesional, construcción del diario de campo, portafolio y producción textual del alumnado en formación. Por lo observado, el camino parece estar fragmentado, incluso antes de que el mismo estudiantado decida seguirlo. Al no comprender el sentido, el estudiantado parece producir como respuesta un discurso carente de información y datos, y como reflexión se puede decir que esta ha sido una práctica profesional desconectada, la cual no articula lo que se aprende en el trayecto de la formación universitaria con lo que se observa en la realidad de las escuelas. 


\section{Referencias}

Barzotto, V. H. y Eufrasio, D. (2009). El informe de práctica docente como manifestación del perfil profesional en Letras (Ariadne C. dos Santos, trad.). Revista MELP (Online), 1, 1-10.

Bolívar, A. (2010). La evaluación de valores y actitudes. McGraw Hill. Fairchild, T. M. (2014). Escritura, saber y conocimiento: Ámbito de investigación en el grado en Letras (Ariadne C. dos Santos, trad.). En Wagner Rodrigues Silva, Janete Silva dos Santos, Márcio Araújo de Melo (Orgs.), Pesquisas em língua(gem) e demandas do ensino básico (23-44). Pontes.

Fairchild, T. M. (2013). Se buscan profesores histéricos: El papel de la escritura en las licenciaturas (Ariadne C. dos Santos, trad.). Estilos da Clínica (USP. Impresso), 18, 71-88.

Freire, P. (1994). Pedagogía del oprimido. Paz e Terra.

Rezende, N. L. (2010). Informe y proyecto en Licenciatura: La escritura como potencialización de la experiencia (Ariadne C. dos Santos, trad.). Linha d'Agua, 23, 47-61.

UPNFM. (2010). Reglamento de Práctica Profesional, Practica Profesional, Capítulo XII. Autor. 